\title{
Takayasu arteritis in an HIV infected woman
}

\author{
Guillermo Ojeda-Burgos ${ }^{1}$, Susana Gómez-Rámirez ${ }^{1}$, Rafael Aguilar-Cuevas' ${ }^{2}$, Manuel \\ Marquez-Solero ${ }^{1}$
}

1. Department of Infectious Diseases, Universitary Hospital Virgen de la Victoria, Málaga, Spain. 2. Department of Radiology, Universitary Hospital Virgen de la Victoria, Málaga, Spain.

Correspondence: Guillermo Ojeda-Burgos. Address: Department of Infectious Diseases, Universitary Hospital Virgen de la Victoria, Málaga, Spain. Email: guilleojeda@gmail.com

Received: January 20, 2014

Accepted: March 11, 2014

Online Published: March 19, 2014

DOI : $10.5430 /$ crim.v1n2p33

URL: http://dx.doi.org/10.5430/crim.v1n2p33

\section{Abstract}

Takayasu arteritis (TA) is a rare chronic vasculitis with particular predilection for the aorta and its major branches. The precipitant factor of the pathological immune response is generally unknown and different factors have been implicated, including human immunodeficiency virus (HIV) infection.

We report the case of a 46 year old woman who complained of a right hemicranial headache for a week and progressive loss of strength in the left side of the body. She was infected by HIV since 1996. After admission she progressed to hyporesponsive and hemiplegia. The absence of brachial pulse, a difference higher than $10 \mathrm{mmHg}$ in the systolic blood pressure between both arms and radiological data were the criteria for the diagnosis of TA and secundary ischemic stroke.

\section{Keywords}

Human immunodeficiency virus, Vasculitis, Autoimmune diseases, Stroke, Vascular diseases

\section{Introduction}

Takayasu arteritis (TA) is a rare chronic vasculitis with particular predilection for the aorta and its major branches. The etiology is unknown, but based on immunohistological findings it is postulated that the inflammatory process is mediated by activation of CD4 $\mathrm{T}$ cell clones that promote pathological chronic inflammatory vascular condition. The precipitant factor of the pathological immune response is generally unknown and different factors have been implicated, including viral infections ${ }^{[1-3]}$. We report a case of TA in a patient infected with human immunodeficiency virus (HIV).

\section{Case presentation}

46 year old female patient who complained of a right hemicranial headache for a week and progressive loss of strength in the left side of the body with deviation of the corner of the mouth. She had knowledge about her HIV infection since 1996 and she had tried different options of antiretroviral therapy (zidovudine, lamivudine, didanosine, stavudine, abacavir, efavirenz, indinavir, fosamprenavir, etravirine) with multiple relapses and fluctuating virological control. Two months earlier she had undetectable HIV viral load and CD4 count of 109/mL. She smoked 10 pack-years and she was carrier of hepatitis C virus (HCV) with most recent viral load undetectable. In 2009 she was diagnosed with squamous vulva cancer 
and treated with radiotherapy and chemotherapy without recurrence known but complicated with radiation secondary enteritis.

Physical examination showed cachexia, bradypsychia, left hemiparesis with supranuclear palsy and gaze deviation to the right, as well as bilateral carotid bruit and right brachial and radial pulse absent. The blood pressure was 175/110 mmHg in the right arm and 160/110 mmHg in the left one. The leukocytes recount at admission was $13.500 / \mathrm{mL}$ and the $\mathrm{C}$ reactive protein (CRP) value was $105 \mathrm{mg} / \mathrm{dL}$. These were the most important findings in the laboratory tests performed. After admission, the patient progressed to hyporesponsive and hemiplegia. A brain TC was performed and it showed multiple hypodense lesions with mass effect in the right cerebellar hemisphere. Temporal, frontal and parietal lobes in the right cerebral hemisphere were affected too. An echo-doppler study of the supra-aortic trunks showed diffuse myointimal thickening around the carotid system and the vertebral arteries with stenosis of the $70 \%$ at the beginning of the right internal carotid artery. An MRI of the brain and supra-aortic trunks with venous contrast confirmed the presence of acute ischemic lesions in the territories of the anterior middle cerebral and right vertebrobasilar arteries (see Figure 1A and 1B), stenosis in the final portion of the both vertebral arteries, right subclavian artery, bifurcation of the right common carotid artery and right external carotid artery as well as severe filiform stenosis in the left common carotid artery with almost complete occlusion of the left internal carotid artery (see Figure 1C and 1D).

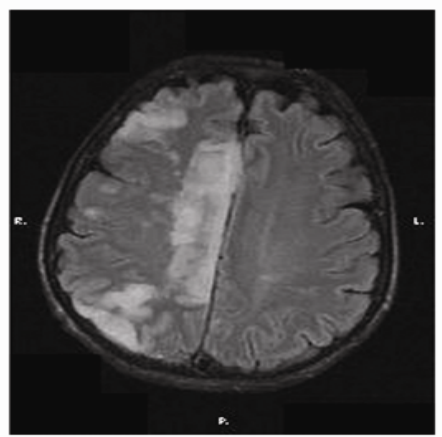

A

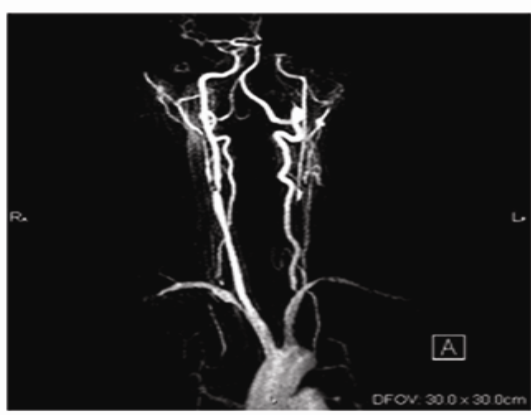

C

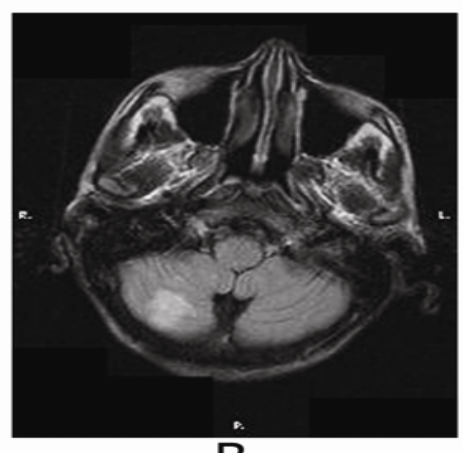

B

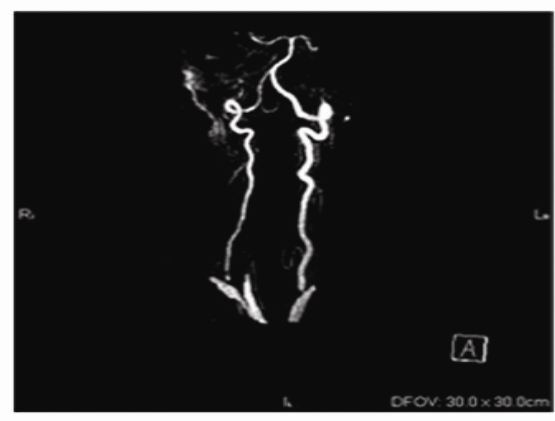

D

Figure 1. MRI of the brain (A, B) and supra-aortic trunks (C, D). It shows T2 hyperintense lesions in the right cerebellar hemisphere and in temporal, frontal and parietal lobes in the right cerebral hemisphere. All of them compatible with acute ischemic lesions (A, B). Axial slices of MRI of supra-aortic trunks that show a stenosis in the output of both vertebral arteries and an affection of the flow in both carotid system and right subclavian artery (C, D).

The study was completed with an abdomen and pelvis CT enhanced with contrast, thrombophilia study and an extensive microbiological study with cultures and serology on blood samples and cerebrospinal fluid to rule out concomitant infections. None of them provided relevant information.

The absence of brachial pulse, a difference higher than $10 \mathrm{mmHg}$ in the systolic blood pressure between both arms and radiological data were the criteria for the diagnosis of TA and secundary ischemic stroke. Treatment was initiated with prednisone at doses of 50mg/day (1mg/kg/day). Antiretrovirals were maintained. The CRP was normalized. The patient 
was discharged with good level of consciousness and left hemiparesis. In three months an angio-CT of the chest and supra-aortic trunks showed the disappearance of the lesions seen in the right carotid system although persisted filiform stenosis at the beginning of the right subclavian artery and an occlusion in the output of the left common carotid.

\section{Discussion}

A great variety of vasculitides, infectious and noninfectious, have been reported in association with HIV infection ${ }^{[4-7]}$. However, large-vessel arteritis in HIV patients is uncommon and usually presents a distinctive pattern of predominantly aneurysmal disease, which can affect the aorta and its branches, and that has been described mainly in subsaharians young adults naive patients ${ }^{[6]}$. This has been attributed mainly to concomitant infectious processes ${ }^{[4,6,8]}$. Two cases of carotid occlusive disease in caucasian males with HIV infection without evidence of opportunistic infection have been published. They were successfully treated with steroids with healing of the stenosis. The authors attribute the process to HIV infection ${ }^{[6,8]}$. The classic large-vessel vasculitis is even less common in patients infected by HIV. In the case of TA we have found only two cases with concomitant HIV infection reported ${ }^{[5,9]}$.

Regardless the precipitant factor considered, the inflammatory process underlying to the TA might be accelerated by different causes of atherosclerosis such as hypertension or tobacco consumption ${ }^{[10]}$. However, HIV itself is a risk factor for atherosclerosis that happens most often and aggressively than in the non-HIV population ${ }^{[11]}$. The use of protease inhibitors (PI) of the first generation can also promote the atherosclerotic changes in the vessels. In the case we report, although the patient was smoker and had received treatment with PI, we believe that the distribution and characteristics of the lesions and the favourable clinical response to steroids point to a systemic inflammatory process vasculitic type with affinity for this region of the vascular tree as more likely cause of this process.

In summary, we report the case of a large-vessel vasculitis with occlusive pattern in a patient infected by HIV that fills criteria for being diagnosed as TA. The HIV infection could help to the development of the inflammatory changes that happen in the vessels of patients with TA. More research is necessary to establish a definitive link between both diseases. Authors declare no conflict of interest regarding to this article.

\section{References}

[1] Sharma BK, Jain S, Sagar S. Systemic manifestations of Takayasu arteritis; the spanding spectrum. Int J Cardiol. $1997 ;$ 54(S):149.

[2] Seko Y, Minota S, Kawasaki A, et al. Perforing secerting killer cell infiltration and expresión of a 65 Kd heat-shock protein in aortic tissue of patients with Takayasu's arteritis. J Clin Invest. 1994; 93: 750. http://dx.doi.org/10.1172/JCI117029

[3] Seko Y, Sato O, Takagi A, et al. Restricted usage of T-cell receptor Valpha-Vbeta genes in infiltrating cells in aortic tissue of patients with Takayasu's arteritis. Circulation. 1996; 93:1788. http://dx.doi.org/10.1161/01.CIR.93.10.1788

[4] Chetty R. Vasculitides associated with HIV infection. J Clin Pathol. 2001; 54: 275-278. http://dx.doi.org/10.1136/jcp.54.4.275

[5] Shingadia D, Das L, Klein-Gitelman M, Chadwick E. Takayasu's arteritis in an human immunodeficiency virus-infected adolescent. Clin Infect Dis. 1999; 29(2): 458-459. http://dx.doi.org/10.1086/520244

[6] Taylor CL,varma A, Herwadkar A, Bonington A. Successful reversal of threatening carotid artery occlusion in HIV-associated non-aneurysmal vasculitis. International Journal of STD \& AIDS. 2008; 19: 141-142. http://dx.doi.org/10.1258/ijsa.2007.007187

[7] Guillevin L. Vasculitides in the context of HIV infection. AIDS. 2008; 22(3): S27-S33. http://dx.doi.org/10.1097/01.aids.0000327513.53255.17

[8] Bermel C, Spüntrup E, Fink G, Nowak DA. Stroke in an adult with VIH infection due to carotid artery stenosis successfully treated with steroids: HIV-associated arteritis?.J Neurol. 2009; 256: 1563-1565. http://dx.doi.org/10.1007/s00415-009-5146-y

[9] Baruteau AE, Martins RP, Boulmier D et al. Acquired left ventricular submitral aneurysms in the course of Takayasu arteritis in a child. Congenit Heart Dis. 2012; 7(1): 76-9. http://dx.doi.org/10.1111/j.1747-0803.2011.00537.x

[10] Seyahi E, Ugurlu S, Cumali R, et al. Atherosclerosis in Takayasu arteritis. Ann Rheum Dis. 2006; 65: 1202. http://dx.doi.org/10.1136/ard.2005.047498

[11] Lozano F. Espectro de enfermedad cardiovascular en pacientes infectados por el VIH. Enferm infecc Microbiol Clin. $2009 ; 27$ : 3-9. http://dx.doi.org/10.1016/S0213-005X(09)73439-1 